\title{
PREVALENCE OF LOW BACKACHE IN PRACTICING DENTISTS OF EASTERN REGION OF NEPAL
}

\author{
Shubham S. ${ }^{1}$, Yadav R.P. ${ }^{2}$
}

\begin{abstract}
AIM: To analyze the prevalence of low backache among practicing dentists of Eastern Region of Nepal.

METHODS: A descriptive cross-sectional, questionnaire based study was conducted among the practicing dentists of Eastern region of Nepal from April 2012 July 2012. A pre-tested, pre-calibrated self-administered verbal rating questionnaire "The Oswestry Low Back Pain Disability Questionnaire" was distributed to a random sample of 85 dentists from different parts of eastern region of Nepal through direct contact and e-mails.
\end{abstract}

RESULTS: A total of 85 dentists were identified among which 76 dentists participated in the research. Research finding showed that the prevalence of back pain among practicing dentists in Eastern region of Nepal was $90.79 \%$ among which $50 \%$ (minimal), 36.84\% (moderate) and 3.95\% (severe).

Among 45 males 4.44\% had no back pain, 53.33\% had minimal, 37.77\% had moderate and 4.44\% had severe back pain. On contrary females (31 participants) showed lesser prevalence of low back pain with $16.12 \%$ no back pain, $45.16 \%$ minimal, $35.48 \%$ moderate and $3.22 \%$ severe back pain.

The participants were divided into 3 different age group intervals in which dentists between 25-30 years were 50 in which $8 \%$ had no back pain, $58 \%$ had minimal, $28 \%$ moderate and $6 \%$ had severe back pain. Between 31-35 years were 22 participants in which 50\% had minimal, 50\% moderate and $0 \%$ had severe low back pain. More than 35 years were 4 participants in which $75 \%$ had no back pain, $25 \%$ had moderate.

Similarly the participants were divided into 5 different groups according the years of clinical practice in dentists having 12 years of clinical practice (37 participants) had $2.70 \%$ no back pain, $56.75 \%$ minimal, 35.13\% moderate and 5.40\% severe back pain. 16 participants had 3-4 years of clinical practice out of which $12.50 \%$ had no low back pain, $62.50 \%$ had minimal, $18.75 \%$ had moderate and $6.25 \%$ had severe back pain.

10 participants had 5-6 years of clinical practice out of which $10 \%$ had no low back pain, 50\% had minimal and $40 \%$ had moderate. 9 participants had 7-8 years of clinical practice out of which $11.11 \%$ had no low back pain, 33.33\% had minimal and $55.55 \%$ had moderate. 5 participants had $>9$ years of clinical practice out of which $75 \%$ had no low back pain, $25 \%$ had minimal and $25 \%$ had moderate.

CONCLUSION: The prevalence of back pain among dental personnel in Eastern region of Nepal was relatively high. The significant risk factors associated with it might be poor posture. Innovations for preventive and, ergonomic strategies should be undertaken to reduce the low back pain.

KEYWORDS: Back pain, Eastern region, Prevalence, Practicing dentist

1. Post Graduate Resident, Department of Conservative and Endodontics, UCMS College of Dental Surgery, Bhairahawa, Nepal

2. Post Graduate Resident, Department of Oral and Maxillofacial Surgery, B.P Koirala Institute of Health Sciences, Dharan, Nepal

\author{
For Correspondence \\ Dr. Snigdha Shubham, \\ Post Graduate Resident, \\ Department of Conservative and Endodontics, \\ UCMS College of Dental Surgery, \\ Bhairahawa, Nepal
}




\section{INTRODUCTION}

Muscular Pain is a common affliction which begins at the time dentists start their professional studies and it stays with them during their professional practice affecting their spine, neck, shoulder and hands. Musculoskeletal pain, particularly low back pain has been found to be a major occupational health hazard in dental profession. At work dentist works in strained postures (both while standing and sitting close to patient) which eventually leads to overstress of back. ${ }^{3}$ Limited working areas, impaired vision associated with oral cavity, long appointments and more concentration requirements during work are some reasons for stressful body positions leading to low back pain. ${ }^{1}$ Low back pain has major health and social implications. Back problems have been cited as the second most common reason for missing work in an industrial population in the United States. ${ }^{1}$ Many studies have been done to determine the prevalence of neck and back problems among dentists and to develop some guidelines to reduce the dental occupational hazards. This study was conducted to assess and increase the level of awareness of occupational hazards, particularly backache among the dentists.

\section{AIM}

Toanalyze theprevalence of low back ache among practicing dentists of Eastern Nepal.

\section{OBJECTIVES}

1. To determine the prevalence of low back pain and its associated factors among practicing dentists of Eastern region of Nepal.

2. To develop some guidelines to reduce the dental occupational hazards.

3. To rate the low back pain disability as minimal, moderate, severe

\section{MATERIAL AND METHODS}

Descriptive cross-sectional, questionnaire based study was done among the practicing dentists of Eastern region of Nepal.

The time period of the study was of 4 months (from April July 2012).

Sample size calculation Formula

\section{Description}

$$
\mathrm{n}=\frac{\mathrm{t}^{2} \times \mathrm{p}(1-\mathrm{p})}{\mathrm{m}^{2}}
$$

$\mathrm{n}=$ required sample size

$\mathrm{t}=$ confidence level at $95 \%$ (standard value of 1.96 )

$\mathrm{p}=$ estimated prevalence of lower back pain in Nepal $(4.9 \%)^{5}$

$\mathrm{m}=$ margin of error at $5 \%$ (standard value of 0.05$)$

On calculation,

The required sample size $=72$.

\section{Inclusion criteria}

Dentists in the Eastern region of Nepal practicing clinical dentistry.

\section{Exclusion criteria}

Dentists not willing to participate in the study.

Dentists with any systemic disease or not able to co-operate.

Dentists not practicing clinical dentistry

\section{Scoring criteria}

The participant selected one statement in each question that most accurately described the effect of his/her pain; if two items were marked, the more severe was scored. Each section was scored on a zero to five scales, with higher values representing greater disability. The sum of the ten scores was expressed as a percentage of the maximum score; this was termed the Oswestry Disability Index (ODI). If the participant failed to complete a section, the percentage score was adjusted (i.e., the score was expressed as a percentage of 45 rather than 50).

ODI percentage scores:
i) Zero
ii) $>$ zero to $\leq 20$
iii) $>20$ to $\leq 40$
iv) $>40$ to $\leq 60$
- no disability
- minimal disability
- moderate disability
- severe disability

Scores exceeding 60 indicated that the patient was severely disabled by pain in several areas of life.

Responses for each question were entered in a spreadsheet program (Microsoft Excel) and the ODI percentage was calculated and expressed as no, minimal, moderate or severe disability.

The calculated ODI percentage was compared according to gender, age of participants and the years of clinical practice.

A total of 85 dentists were distributed questionnaire via direct contact and e-mail, out of which 76 responded and were analyzed.

\section{Chart 1: Prevalence of low back pain}

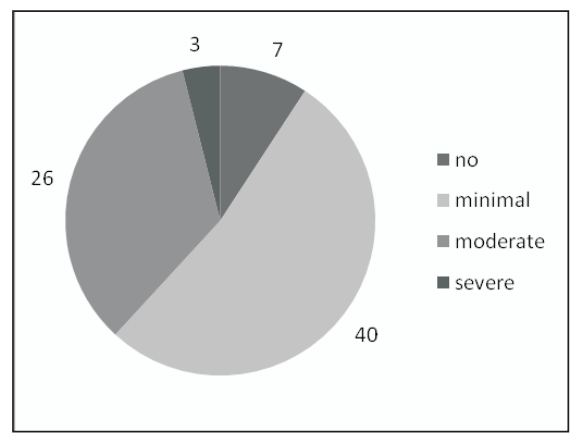




\section{Chart 2: Prevalence of low back pain according to gender}

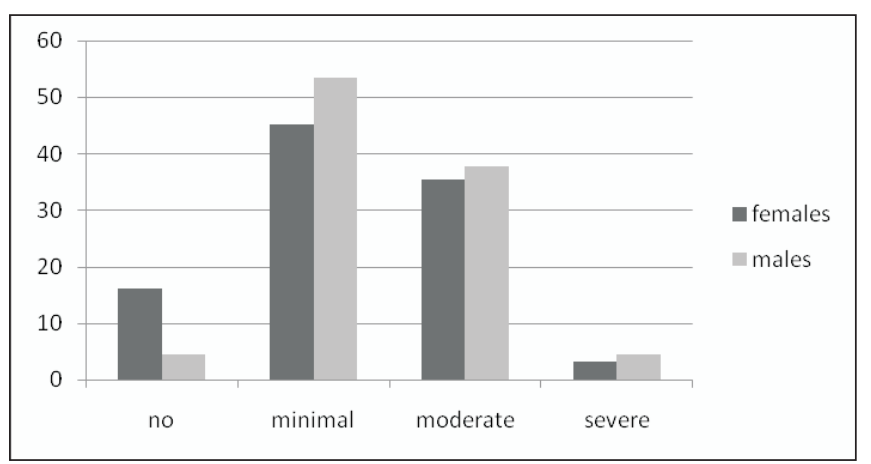

Chart 3: Prevalence of low back pain according to age

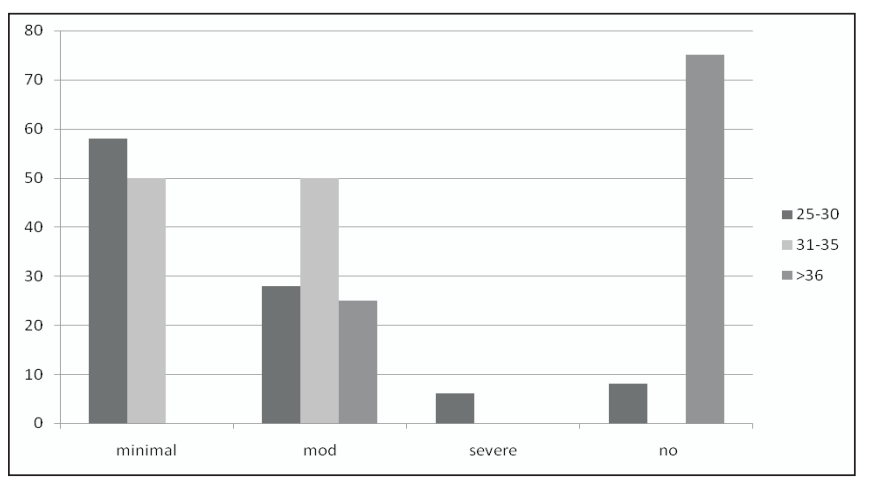

Chart 4: Prevalence of low back pain according to years of clinical practice

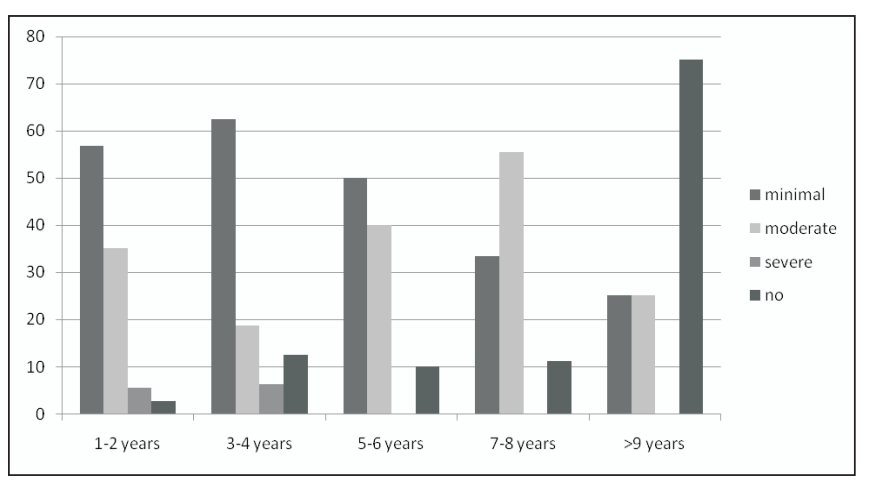

The study was conducted with the purpose to find out the prevalence of low back pain among the practicing dentists in Eastern Region of Nepal. A total of about 85 dentists were identified among which 76 dentists participated in the research. Some of the reasons for excluding remaining dentists from the study were direct refusals, move on to other places and attrition.

Research finding showed that the prevalence of back pain among practicing dentists in Eastern region of Nepal was 50\% (minimal), 36.84\% (moderate) and 3.95\% (severe). The finding is comparable to that of Sweden (43\%) and some other Asian countries such as Thailand (50\%) and Hong Kong (43\%) but differ markedly from $28 \%$ prevalence reported in Finland.

Findings revealed that low back pain were more prevalent in males than in females and with the increase in age and clinical experience, the low back pain was found to be less prevalent.

Conducive to our study, a research done by Wilder and Pope, 1996 has placed males at a greater risk of developing low back pain. In the study done by Callaghan and Dunk, 2002, males adopted lumbar spine angles that were well within the range for flexion relaxation whereas female spine angles were at the lower margin of this range. Flexion relaxation has been proposed as a mechanism for low back pain during sitting through potential stretching of the passive tissues responsible for bearing the load moment after the muscles shut off, further emphasizing the importance of maintaining near neutral postures. The typical female sitting posture documented in their study demonstrated that females tend to avoid excessive spine flexion and maintain a more upright posture thus leading to less prevalence of low back pain as compared to males.

Similarly the result showing a decrease in prevalence of low back pain with increase in age and clinical experience in our study is consistent with the study done by Leggat et al (2007). They reported that back pain was more common among younger Australian dentists. This finding could be attributed to work inexperience and inadequate knowledge in dental procedures among the younger dentists. In this regard, an investigation of Thai dentists revealed that less experienced dentists were more likely to suffer from musculoskeletal pain than their more experienced counterparts. Possible explanations were that experienced dentists are probably better at adjusting their working position and techniques in order to avoid musculoskeletal problems compared to their less experienced counterparts, or they simply developed coping strategies to deal with the pain.

\section{CONCLUSIONS}

It can be concluded that the prevalence of low back pain among dentists in Eastern Region of Nepal is high. Low back pain remains a major occupational health problem for practicing dentists. Younger dentists had more symptoms than the older dentists. The male dentists had relatively higher frequency of pain than their female counterparts.

Knowledge about the scope of ergonomics and prophylaxis, as well as health and safety measures at the workplace are important to prevent the risk of developing back pain.

\section{LIMITATIONS}

It must be stated here that causal inferences cannot be drawn from the results of this cross-sectional study. It is common knowledge that causal relationships need to be evaluated by a cohort study. 
Another limitation of this study was the reliance on selfreported data. A healthy worker effect may also pose a limitation to this study as dentists who had left dental practice due to back pain were not included in the study. The study can be conducted in larger sample to generalize the result. Longitudinal study can be conducted to identify the actual relationship of different socio-demographic factors with low back pain.

Similar kind of study can be done to assess prevalence of other musculoskeletal disorders among the dentists apart from low back pain

\section{RECOMMENDATIONS}

In order to reduce low back pain problem, correct postural practices, relaxation interval sessions during work, weight monitoring, preventive and, ergonomic strategies as well as specific therapeutic programs might be carried out to tackle the identified risk factors.

\section{ACKNOWLEDGEMENT}

We take this opportunity to express our sincere gratitude to our respected seniors Dr Blaise Antony and Dr Ravish Mishra for their warm, comforting, inspiring nature and of course constant supervision and guidance without whom this article would not have been possible.

We are both obligated and pleased to thank B.P.Koirala Institute of Health Sciences (BPKIHS) authority and Department of Community Dentistry, BPKIHS for providing us an opportunity and facilities to conduct this research. We are also thankful to Dr. Ashish Shrestha, Associate proffesor, BPKIHS for his support.
We express our sincere gratitude to all the practising dentists of eastern region of Nepal for their enthusiastic and active participation in completion of this study.

\section{REFERENCES}

1. Rundcrantz BL. Pain and Discomfort in the Musculoskeletal System among Dentists. Sweden J Suppl. 1991;76:101-02.

2. Dajpratham P, Ploypetch T,Kiattavorncharoen S, Boonsiriseth $K$. Prevalence and associated factors of musculoskeletal pain among the dental personnel in a dental school. JMed Assoc Thai. 2010;93(6):714-21.

3. Li TKL, Lo ECM, Wong AH, Wai-Hung MWH, Leung JL. SelfReported Occupation-Related Health Problems in Hong Kong Dentists. Hong Kong Dental Journal. 2007;3:39-44.

4. Kerosuo E, Kerosuo H, Kanerva L. Self-Reported Health Complaints among General Dental Practitioners, Orthodontists and Office Employees. Acta Odontol Scand. 2000;58:207-12.

5. Wilder DG, Pope MH, 1996. Epidemiological and aetiological aspects of low back pain in vibration environmentsan update.Clin. Biomech. 11, 61-73.

6. Callaghan JP, Dunk NM, 2002. Examination of the flexion relaxation phenomenon in erector spinae muscles during short duration slumped sitting. Clin. Biomech. 17, 353-60.

7. Leggat PA, Smith DR. Musculoskeletal disorders selfreported by dentists in Queensland, Australia. Aust Dent J 2006;51(4):324-27.

8. Chowanadisai S, Kukiattrakoon B, Yapong B, Kedjarune U, Leggat PA. Occupational health problems of dentists in southern Thailand. Int Dent J 2000;50:36-40. 